\title{
A new conjugate gradient algorithms using conjugacy condition for solving unconstrained optimization
}

\author{
Aseel M. Qasim', Zinah F. Salih², Basim A. Hassan ${ }^{3}$ \\ ${ }^{1}$ Department of Mathematics, College of Education of Pure Sciences, University of Mosul, Nineveh, Iraq \\ ${ }^{2,3}$ Department of Mathematics, College of Computers Sciences and Mathematics, University of Mosul, Nineveh, Iraq
}

\section{Article Info}

\section{Article history:}

Received May 28, 2021

Revised Oct 14, 2021

Accepted Oct 27, 2021

\section{Keywords:}

Conjugacy condition

Conjugate gradient method

Global convergence

Sufficient descent property

\begin{abstract}
The primarily objective of this paper which is indicated in the field of conjugate gradient algorithms for unconstrained optimization problems and algorithms is to show the advantage of the new proposed algorithm in comparison with the standard method which is denoted as. Hestenes Stiefel method, as we know the coefficient conjugate parameter is very crucial for this reason, we proposed a simple modification of the coefficient conjugate gradient which is used to derived the new formula for the conjugate gradient update parameter described in this paper. Our new modification is based on the conjugacy situation for nonlinear conjugate gradient methods which is given by the conjugacy condition for nonlinear conjugate gradient methods and added a nonnegative parameter to suggest the new extension of the method. Under mild Wolfe conditions, the global convergence theorem and lemmas are also defined and proved. The proposed method's efficiency is programming and demonstrated by the numerical instances, which were very encouraging.
\end{abstract}

This is an open access article under the CC BY-SA license.

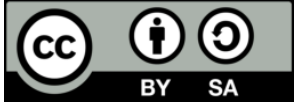

\section{Corresponding Author:}

Zinah F. Salih

Department of Mathematics, College of Computers Sciences and Mathematics

University of Mosul

Mosul, 41001, Nineveh, Iraq

Email: zn_f2020@uomosul.edu.iq

\section{INTRODUCTION}

The conjugate gradient method is a group of very important smooth function minimization methods, which is have a large dimension. We are interesting with conjugate gradient method for locating the function's local minimum,

$$
\min \left\{f(x) \mid x \in R^{n}\right\}
$$

where $f: R^{n} \rightarrow R^{1}$ is a nonlinear, smooth function whose gradient is denoted by $g_{x}=\nabla f\left(x_{k}\right)$, the iteration method that the conjugate gradient $(\mathrm{CG})$ method uses in it of the line search is:

$$
x_{0} \in R^{n}, \quad x_{k+1}=x_{k}+\alpha_{k} d_{k}
$$

where $\alpha_{k}$ is the step size, in order to ensure the new method's global convergence, we will impose that the step length $\alpha_{k}$ satisfies the Wolfe conditions which have a good property to prove convergence for more detail see [1]-[6]: 


$$
\begin{aligned}
& f\left(x_{k}+\alpha_{k} d_{k}\right)-\mathrm{f}\left(x_{k}\right) \leq \sigma_{1} \alpha g_{k}^{T} d_{k} \\
& g\left(x_{k}+\alpha_{k} d_{k}\right)^{T} \geq \sigma_{2} g_{k}^{T} d_{k}
\end{aligned}
$$

where $0<\sigma_{1}<\sigma_{2}<1$ see [7] and $d_{k}$ is the search direction generation by:

$$
d_{k+1}=-g_{k+1}+\beta_{k} d_{k}
$$

where $\beta_{k}$ is the CG update parameter, which is a scalar. It's worth noting that the formula specification of the update parameter is a critical component of any CG algorithm, which is why different CG algorithms have been proposed to conform to different choices of $\beta_{k}$ in (5). In [8]-[13], the parameters are given by:

$$
\begin{aligned}
& \beta_{k}^{F R}=\frac{g_{k+1}^{T} g_{k+1}}{g_{k}^{T} g_{k}} \\
& \beta_{k}^{D Y}=\frac{g_{k+1}^{T} g_{k+1}}{y_{k}^{T} d_{k}} \\
& \beta_{k}^{P R P}=\frac{g_{t+1}^{T} y_{k}}{\left\|g_{k}\right\|^{2}} \\
& \beta_{k}^{H S}=\frac{g_{k+1}^{T} g_{k+1}}{d_{k}^{T} y_{k}} \\
& \beta_{k}^{B S Q}=\frac{g_{k+1}^{T} g_{k+1}}{\alpha\left(g_{k}^{T} d_{k}\right)^{2} / 2\left(f_{k}-f_{k+1}\right)}
\end{aligned}
$$

Furthermore, some CG methods for unconstrained optimization problem are not globally convergent. As a result, the researchers have been working to improve CG methods that are both globally convergent and numerically efficient [14]. We present the motivation and new algorithm of the proposed method. The global convergence of the proposed algorithm is exhibited with distinct quite different computational efficiency.

\section{THE PROPOSED METHOD}

The conjugacy condition for nonlinear conjugate gradient methods is given by (11).

$$
y_{k}^{T} d_{k+1}=0
$$

Perry decided to extend the deadline of (11) as:

$$
d_{k+1}^{T} y_{k}=-g_{k+1}^{T} s_{k}
$$

Dai and Liao in [15] took Perry's approach and added a nonnegative parameter $t$ to suggest the following extension of (12).

$$
d_{k+1}^{T} y_{k}=-t g_{k+1}^{T} s_{k}
$$

We suggest this modification to the numerator of $\beta_{k}^{B S Q}$ [13], update the parameter to obtain:

$$
\beta_{\mathrm{k}}^{\mathrm{MBSQ}}=\frac{\left\|\mathrm{g}_{\mathrm{k}+1}\right\|^{2}+\operatorname{tg}_{\mathrm{k}+1}^{\mathrm{T}} \mathrm{s}_{\mathrm{k}}}{\alpha\left(\mathrm{g}_{\mathrm{k}}^{\mathrm{T}} \mathrm{d}_{\mathrm{k}}\right)^{2} / 2\left(\mathrm{f}_{\mathrm{k}}-\mathrm{f}_{\mathrm{k}+1}\right)}
$$

Following the Li et al. approach in [16] and $\beta_{k}^{M B S Q}$ we modified the following parameter extension:

$$
\beta_{k}^{M B S Q}=\frac{\left\|g_{k+1}\right\|^{2}}{\alpha\left(g_{k}^{T} d_{k}\right)^{2} / 2\left(f_{k}-f_{k+1}\right)}+t \frac{g_{k+1}^{T} s_{k}}{\alpha\left(g_{k}^{T} d_{k}\right)^{2} / 2\left(f_{k}-f_{k+1}\right)}=\beta_{k}^{B S Q}+t \frac{g_{k+1}^{T} s_{k}}{\alpha\left(g_{k}^{T} d_{k}\right)^{2} / 2\left(f_{k}-f_{k+1}\right)}
$$


where $\mathrm{t}$ is a nonnegative parameter, whose values have been calculated by the use of the conjugacy condition analysis. More of than we compute t numerically by multiplying search direction by $y_{k}$ and by using (14) we obtain:

$$
y_{k}^{T} d_{k+1}=-y_{k}^{T} g_{k+1}+\beta_{k}^{M B S Q} y_{k}^{T} d_{k}
$$

Now we substitute $y_{k}^{T} d_{k+1}=-t g_{t+1}^{T} s_{k}$ if the direction is in exact (ILS) and so we have:

$$
\begin{aligned}
& -t g_{k+1}^{T} s_{k}=-y_{k}^{T} g_{k+1}+\frac{g_{k+1}^{T} g_{k+1}+t g_{k+1}^{T} s_{k}}{\alpha\left(g_{k}^{T} d_{k}\right)^{2} / 2\left(f_{k}-f_{k+1}\right)} y_{k}^{T} d_{k} \\
& -t g_{k+1}^{T} s_{k}-\frac{\left(t g_{k+1}^{T} s_{k}\right) y_{k}^{T} d_{k}}{\alpha\left(g_{k}^{T} d_{k}\right)^{2} / 2\left(f_{k}-f_{k+1}\right)}=-y_{k}^{T} g_{k+1}+\frac{\left(g_{k+1}^{T} g_{k+1}\right) y_{k}^{T} d_{k}}{\alpha\left(g_{k}^{T} d_{k}\right)^{2} / 2\left(f_{k}-f_{k+1}\right)} \\
& t=\frac{y_{k}^{T} g_{k+1}-\frac{g_{k+1}^{T} g_{k+1} y_{k}^{T} d_{k}}{\alpha\left(g_{k}^{T} d_{k}\right)^{2} / 2\left(f_{k}-f_{k+1}\right)}}{g_{k+1}^{T} s_{k}+\frac{g_{k+1} y_{k}^{T} d_{k}}{\alpha\left(g_{k}^{T} d_{k}\right)^{2} / 2\left(f_{k}-f_{k+1}\right)}} \\
& t=\frac{\left(y_{k}^{T} g_{k+1}\right) \alpha\left(g_{k}^{T} d_{k}\right)^{2} / 2\left(f_{k}-f_{k+1}\right)-g_{k+1}^{T} g_{k+1} y_{k}^{T} d_{k}}{g_{k+1}^{T} s_{k} \alpha\left(g_{k}^{T} d_{k}\right)^{2} / 2\left(f_{k}-f_{k+1}\right)+g_{k+1}^{T} s_{k} y_{k}^{T} d_{k}}
\end{aligned}
$$

Substituting the value of $\mathrm{t}$ from (17) into (15) yields:

$$
\begin{aligned}
\beta_{k}^{M S Q} & =\frac{g_{k+1}^{T} g_{k+1}+t g_{k+1}^{T} s_{k}}{\alpha\left(g_{k}^{T} d_{k}\right)^{2} / 2\left(f_{k}-f_{k+1}\right)} \\
& =\frac{g_{k+1}^{T} g_{k+1}+\frac{\left(y_{k}^{T} g_{k+1}\right) \alpha\left(g_{k}^{T} d_{k}\right)^{2} / 2\left(f_{k}-f_{k+1}\right)-g_{k+1}^{T} g_{k+1} y_{k}^{T} d_{k}}{g_{k+1}^{T} s_{k} \alpha\left(g_{k}^{T} d_{k}\right)^{2} / 2\left(f_{k}-f_{k+1}\right)+g_{k+1}^{T} s_{k} y_{k}^{T} d_{k}} s_{k+1}}{\alpha\left(g_{k}^{T} d_{k}\right)^{2} / 2\left(f_{k}-f_{k+1}\right)}
\end{aligned}
$$

By taking $g_{k+1}^{T} s_{k}$ from the numerator and denominator as common factor:

$$
\begin{aligned}
& \frac{g_{k+1}^{T} g_{k+1}+\frac{\left(y_{k}^{T} g_{k+1}\right) \alpha\left(g_{k}^{T} d_{k}\right)^{2} / 2\left(f_{k}-f_{k+1}\right)-g_{k+1}^{T} g_{k+1} y_{k}^{T} d_{k}}{\alpha\left(g_{k}^{T} d_{k}\right)^{2}}+y_{k}^{T} d_{k}}{\alpha\left(g_{k}^{T} d_{k}\right)^{2} / 2\left(f_{k}-f_{k+1}\right)} \\
&= \frac{g_{k+1}^{T} g_{k+1}\left(\frac{\alpha\left(g_{k}^{T} d_{k}\right)^{2}}{2\left(f_{k}-f_{k+1}\right)}+y_{k}^{T} d_{k}\right)+\left(y_{k}^{T} g_{k+1}\right) \alpha\left(g_{k}^{T} d_{k}\right)^{2} / 2\left(f_{k}-f_{k+1}\right)-g_{k+1}^{T} g_{k+1} y_{k}^{T} d_{k}}{\left[\frac{\alpha\left(g_{k}^{T} d_{k}\right)^{2}}{2\left(f_{k}-f_{k+1}\right)}+y_{k}^{T} d_{k}\right] \alpha\left(g_{k}^{T} d_{k}\right)^{2} / 2\left(f_{k}-f_{k+1}\right)} \\
&= \frac{g_{k+1}^{T} g_{k+1}\left(\frac{\alpha\left(g_{k}^{T} d_{k}\right)^{2}}{2\left(f_{k}-f_{k+1}\right)}+y_{k}^{T} d_{k}-y_{k}^{T} d_{k}\right)+\left(y_{k}^{T} g_{k+1}\right) * \frac{\alpha\left(g_{k}^{T} d_{k}\right)^{2}}{2\left(f_{k}-f_{k+1}\right)}}{\left[\frac{\alpha\left(g_{k}^{T} d_{k}\right)^{2}}{2\left(f_{k}-f_{k+1}\right)}+y_{k}^{T} d_{k}\right] * \frac{\alpha\left(g_{k}^{T} d_{k}\right)^{2}}{2\left(f_{k}-f_{k+1}\right)}} \\
& \beta_{k}^{M S Q}=\frac{g_{k+1}^{T} g_{k+1}+y_{k}^{T} g_{k+1}}{\frac{\alpha\left(g_{k}^{T} d_{k}\right)^{2}}{2\left(f_{k}-f_{k+1}\right)^{T}}+y_{k}^{T} d_{k}}
\end{aligned}
$$

\subsection{Outlines of the new algorithm}

Define $\mathrm{k}=0$ and choose an initial point $x_{0} \in R^{n}$

If $\left\|g_{k}\right\|=0$, then terminate, otherwise proceed next step.

Calculate the descent $d_{k}$ using (5). 
Determine a step size $\alpha_{k}$ by Wolfe line search condition (3), (4).

Let $x_{k+1}=x_{k}+\alpha_{k} d_{k}$.

Estimate $\beta_{k}$ which defined in (18).

Set $\mathrm{k}=\mathrm{k}+1$ and proceed to step (2).

\section{GLOBAL CONVERGENCE}

The following assumption is needed to analyze the algorithm's global convergence.

a) The level set $\xi=\left\{x \in R^{n} \mid f(x) \leq f\left(x_{1}\right\}\right.$ is bounded.

b) $\mathrm{f}(\mathrm{x})$ is continuously differentiable and its gradient is Lipchitz continuous in some neighborhood $\mathrm{u}$ of $\xi$, that is to say in particular, there exists a constant $p>0$ such that $\|g(x)-g(y)\| \leq\|x-y\| \forall x, y \epsilon \xi$, more details can be found in [17], The well-known Zoutendijk condition [18] is given by the following lemma.

\subsection{Lemma (1)}

Assume that assumptions a) and b) are true, and consider any iteration of the form (5), where $d_{k}$ is the path direction and the step size $\alpha_{k}$ satisfies the condition (3), (4), then the zoutendijk condition:

$$
\sum_{k=1}^{\infty} \frac{\left(g_{k}^{T} d_{k}\right)^{2}}{\left\|d_{k}\right\|^{2}}<\infty
$$

holds.

\subsection{Lemma (2)}

If $d_{k+1}$ is given in (5) and $\beta_{k}^{M S Q}$ is given in (18), the following result holds.

$$
g_{k+1}^{T} d_{k+1}<-c\left\|g_{k+1}\right\|^{2} \forall k
$$

Proof:

We have $d_{1}=-g_{1}$ then $d_{1}^{T} g_{1}<0$ this is by induction for $\mathrm{k}=1$, then we conclude that $d_{k}^{T} g_{k}<$ $0, \forall k \geq 2,[19]$.

$$
g_{k+1}^{T} d_{k+1}=-\left\|g_{k+1}\right\|^{2}+\frac{g_{k+1}^{T} g_{k+1}+y_{k}^{T} g_{k+1}}{\alpha\left(g_{k}^{T} d_{k}\right)^{2} / 2\left(f_{k}-f_{k+1}\right)+y_{k}^{T} d_{k}} g_{k+1}^{T} d_{k}
$$

In [8], It follows from $\left|g_{k+1}^{T} d_{k}\right| \leq-\sigma g_{k}^{T} d_{k}$ that $g_{k+1}^{T} d_{k} \leq-\sigma g_{k}^{T} d_{k}$ and $d_{k}^{T} y_{k}=d_{k}^{T}\left(g_{k+1}-g_{k}\right) \geq(\sigma-$ 1) $g_{k}^{T} d_{k}$ and from $d_{k}^{T} g_{k} \leq-c\left\|g_{k}\right\|^{2}$ by using powell restart equation (i.e $\left|g_{k}^{T} g_{k+1}\right| \geq 0.2 g_{k+1}^{2}$ )

$$
\begin{aligned}
& g_{k+1}^{T} d_{k+1} \leq-\left\|g_{k+1}\right\|^{2}+\frac{\left(\left\|g_{k+1}\right\|^{2}+\left\|g_{k+1}\right\|^{2}\right)\left(-\sigma g_{k}^{T} d_{k}\right)}{\alpha\left(g_{k}^{T} d_{k}\right)^{2} / 2\left(f_{k}-f_{k+1}\right)+(\sigma-1) g_{k}^{T} d_{k}} \\
& \leq-\left\|g_{k+1}\right\|^{2}+\frac{-2\left\|g_{k+1}\right\|^{2} \sigma}{\left(\alpha\left(g_{k}^{T} d_{k}\right) / 2\left(f_{k}-f_{k+1}\right)\right)+(\sigma-1)}
\end{aligned}
$$

From (3) and (4) we have

$$
\begin{aligned}
& g_{k+1}^{T} d_{k+1} \leq-\left\|g_{k+1}\right\|^{2}+\frac{-2 \sigma\left\|g_{k+1}\right\|^{2} 2\left(f_{k}-f_{k+1}\right)}{\alpha\left(g_{k}^{T} d_{k}\right)+(\sigma-1)\left(2\left(f_{k}-f_{k+1}\right)\right)} \\
& \leq-\left\|g_{k+1}\right\|^{2}+\frac{2 \sigma\left\|g_{k+1}\right\|^{2} 2\left(f_{k+1}-f_{k}\right)}{\alpha\left(g_{k}^{T} d_{k}\right)+(\sigma-1)\left(2\left(f_{k}-f_{k+1}\right)\right)} \\
& \leq-\left\|g_{k+1}\right\|^{2}+\frac{2 \sigma\left\|g_{k+1}\right\|^{2}\left(2 \delta \alpha g_{k} d_{k}\right)}{\alpha\left(g_{k}^{T} d_{k}\right)+(\sigma-1)\left(-2 \delta \alpha g_{k} d_{k}\right)} \\
& \leq-\left\|g_{k+1}\right\|^{2}+\frac{2 \sigma\left\|g_{k+1}\right\|^{2}(2 \delta)}{1+(\sigma-1)(-2 \delta)}
\end{aligned}
$$


Let $\ell=\frac{2 \sigma(2 \delta)}{1+(\sigma-1)(-2 \delta)}$ and $0<\ell<1$, then we have $g_{k+1}^{T} d_{k+1} \leq-\left\|g_{k+1}\right\|^{2}+\ell\left\|g_{k+1}\right\|^{2}$

$\therefore g_{k+1}^{T} d_{k+1} \leq-c\left\|g_{k+1}\right\|^{2}$

By this mathematical induction, we reach the result.

\subsection{Lemma (3)}

Assume that $x_{1}$ is a starting point for which assumption a) is hold. If the new method generates $x_{1}, x_{2}, x_{3}$, and so on then,

$$
\lim _{k \rightarrow \infty} \inf \left\|g_{k}\right\|=0
$$

Proof: Assume that the inference is false, that is, there is an appositive constant such that $\left\|g_{k}\right\| \geq \rho$ for all $\mathrm{k}$, since

$$
\begin{aligned}
& d_{k+1}=-g_{k+1}+\beta_{k} d_{k} \text { which can be written as } \\
& d_{k+1}+g_{k+1}=\beta_{k} d_{k}
\end{aligned}
$$

and since

$$
\begin{aligned}
& \beta_{k}=\frac{g_{k+1}^{T} g_{k+1}+y_{k}^{T} g_{k+1}}{\alpha\left(g_{k}^{T} d_{k}\right)^{2} / 2\left(f_{k}-f_{k+1}\right)+y_{k}^{T} d_{k}} \text { and from Wolfe condition (3), (4) } \\
& f_{k}-f_{k+1} \geq-\delta \alpha g_{k}^{T} d_{k} \text { and from } d_{k}^{T} y_{k} \geq(\partial-1) g_{k}^{T} d_{k}[8] \\
& \therefore \beta_{k} \leq \frac{2\left\|g_{k+1}\right\|^{2} \quad .2\left(f_{k}-f_{k+1}\right)}{\alpha\left(g_{k}^{T} d_{k}\right)^{2}+2 y_{k}^{T} d_{k}\left(f_{k}-f_{k+1}\right)} \\
& \leq \frac{2\left\|g_{k+1}\right\|^{2} .2\left(f_{k}-f_{k+1}\right)}{\alpha\left(g_{k}^{T} d_{k}\right)^{2}+2 y_{k}^{T} d_{k}\left(-\delta \alpha g_{k}^{T} d_{k}\right)} \\
& \leq \frac{2\left\|g_{k+1}\right\|^{2} \cdot(-2)\left(f_{k+1}-f_{k}\right)}{\alpha\left(g_{k}^{T} d_{k}\right)^{2}+2 y_{k}^{T} d_{k}\left(-\delta \alpha g_{k}^{T} d_{k}\right)} \\
& \leq \frac{2\left\|g_{k+1}\right\|^{2} \cdot-\left(2 \alpha \delta g_{k}^{T} d_{k}\right)}{\alpha\left(g_{k}^{T} d_{k}\right)^{2}+2(\sigma-1) g_{k}^{T} d_{k}\left(-\delta \alpha g_{k}^{T} d_{k}\right)} \\
& \leq \frac{-4\left\|g_{k+1}\right\|^{2} \cdot \alpha \delta}{g_{k}^{T} d_{k}[\alpha+2(\sigma-1)-\delta \alpha]} \\
& \leq \frac{-4\left\|g_{k+1}\right\|^{2} \cdot . \alpha \delta}{-\left\|g_{k}\right\|^{2}[\alpha+2(\sigma-1)-\delta \alpha]} \\
& \therefore\left|\beta_{k}\right|<j \text { such that } \mathrm{j} \text { is constant } \\
& \left\|d_{k+1}\right\|=\left\|-g_{k+1}\right\|+\left|\beta_{k}\right|\left\|d_{k}\right\| \\
& \leq\left\|-g_{k+1}\right\|+|j|\left\|d_{k}\right\| \\
& \leq £+|j| \ell
\end{aligned}
$$

and we concluding the proof with this contradiction that is:

$$
\sum_{i=1}^{k} \frac{1}{\left\|d_{k}\right\|^{2}} \geq \frac{1}{(€+|j| \ell)^{2}} \sum 1=\infty
$$

Which is contradiction with zoutendijk theorem therefore the algorithm is globally convergent. 


\section{RESULT AND DISCUSSION}

Now, we preset the arithmetical experiments we test and compare our method with $B_{k}^{H S}$, the code is written in using Fortran 90 to apply these methods and the test function are selected from [20]. The stopping state is defined as $\left\|g_{k+1}\right\| \leq 10^{-6}$ as recommended by [21]. We used 14 test problems with dimension 100 and 1000. The computation results shown in Table 1 have the following meaning: the total number of iterations (NOI), the total number of restarts (NOR), and the total number of function evaluations (NOF). Also, Table 2 shows the average efficiency of the new algorithm with respect to harmony search (HS) method. Furthermore, optimization problems used in many papers for example, see [22]-[25].

Table 1. Numerical results of new algorithms and HS-CG algorithm

\begin{tabular}{lccccccc}
\hline Test Function & $\mathrm{n}$ & \multicolumn{3}{c}{ New Formula } & \multicolumn{3}{c}{ HS } \\
& & NOI & NOR & NOF & NOI & NOR & NOF \\
\hline Quadratic Diagonal Perturbed & 100 & 48 & 11 & 86 & 52 & 10 & 90 \\
& 1000 & 167 & 31 & 291 & 171 & 36 & 302 \\
Trigonometric & 100 & 18 & 9 & 34 & 19 & 10 & 36 \\
& 1000 & 38 & 22 & 68 & 33 & 19 & 64 \\
Extended Beale & 100 & 12 & 7 & 24 & 14 & 8 & 27 \\
Extended Himmelblau & 1000 & 14 & 9 & 27 & 15 & 10 & 29 \\
& 100 & 10 & 6 & 19 & 10 & 6 & 19 \\
HIMMELBH (CUTE) & 1000 & 10 & 6 & 19 & 11 & 6 & 21 \\
& 100 & 6 & 3 & 13 & 6 & 3 & 13 \\
Broyden Tridiagonal & 1000 & 6 & 3 & 13 & 6 & 3 & 13 \\
Almost Perturbed Quadratic & 100 & 29 & 6 & 50 & 28 & 5 & 50 \\
EDENSCH (CUTE) & 1000 & 34 & 13 & 59 & 38 & 17 & 61 \\
DIXON3DQ (CUTE) & 1000 & 283 & 78 & 448 & 346 & 106 & 547 \\
& 100 & 28 & 10 & 51 & 38 & 22 & 466 \\
DENSCHNA (CUTE) & 1000 & 34 & 18 & 230 & 44 & 27 & 617 \\
DENSCHNC (CUTE) & 100 & 432 & 120 & 689 & 487 & 139 & 762 \\
DENSCHNB (CUTE) & 1000 & 2001 & 551 & 3181 & 2001 & 543 & 3160 \\
DENGSB1 (CUTE) & 100 & 9 & 6 & 17 & 9 & 6 & 17 \\
Total & 1000 & 9 & 6 & 18 & 9 & 6 & 18 \\
& 100 & 14 & 9 & 27 & 13 & 9 & 24 \\
& 1000 & 4 & 10 & 27 & 12 & 9 & 24 \\
& 100 & 7 & 5 & 15 & 7 & 5 & 15 \\
& 1000 & 7 & 5 & 15 & 7 & 5 & 15 \\
& 100 & 26 & 22 & 42 & 24 & 19 & 41 \\
& 1000 & 20 & 16 & 37 & 28 & 23 & 47 \\
& 100 & 423 & 116 & 673 & 551 & 156 & 868 \\
& 1000 & 2001 & 552 & 3155 & 2001 & 579 & 3155 \\
& & 5780 & 1675 & 9469 & 6092 & 1827 & 10676 \\
\hline & & & & & & &
\end{tabular}

Table 2. Average efficiency of the new algorithm

\begin{tabular}{rcc}
\hline & HS algorithm & New Formula algorithm \\
\hline NOI & $100 \%$ & $94.88 \%$ \\
NOR & $100 \%$ & $91.68 \%$ \\
NOF & $100 \%$ & $88.69 \%$ \\
\hline
\end{tabular}

\section{CONCLUSION}

A new CG-method based on the conjugacy condition have been derived with a new update parameter, which is demonstrated the theory of global convergence for one of the proposed methods under reasonable assumptions. According to numerical results, the proposed algorithms has outperformed the regular HS method on average. Furthermore, the efficiency of new algorithm has been explained clearly based on arithmetic results.

\section{ACKNOWLEDGEMENTS}

The authors are grateful to the University of Mosul/College of Computer Sciences and Mathematics for providing facilities that aided in the production of this paper.

\section{REFERENCES}

[1] P. Wolfe, "Convergence conditions for ascent methods," SIAM review, vol. 11, no. 2, pp. 226-235, 1969, doi: $10.1137 / 1011036$. 
[2] J. Wang and X. Chi, "CG global convergence properties with Goldstein linesearch," Bulletin of the Brazilian Mathematical Society, vol. 36, no. 2, pp. 197-204, 2005, doi: 10.1007/s00574-005-0036-0.

[3] S. Babaie-Kafaki and R. Ghanbari, "An optimal extension of the Polak-Ribière-Polyak conjugate gradient method," Numerical Functional Analysis and Optimization, vol. 38, no. 9, pp. 1115-1124, 2017, doi: 10.1080/01630563.2017.1320673.

[4] Z. Wei, S. Yao, and L. Liu, "The convergence properties of some new conjugate gradient methods," Applied Mathematics and computation, vol. 183, no. 2, pp. 1341-1350, 2006, doi: 10.1016/j.amc.2006.05.150.

[5] X. L. Dong, H. W. Liu, Y. B. He, S. Babaie-Kafaki, and R. Ghanbari, "A New Three-Term Conjugate Gradient Method with Descent Direction for Unconstrained Optimization," Mathematical Modelling and Analysis, vol. 21, no. 3, pp. 399-411, 2016, doi: 10.3846/13926292.2016.1176965.

[6] Y. H. Dai and Y. Yuan, "Further studies on the Polak-Ribiere-Polyak method," Institute of Computational Mathematics and Scientific Engineering Comput-ing, Chinese Academy of Sciences, Report ICM-95-040, 1995.

[7] M. Li, H. Liu, and Z. Liu, "A new family of conjugate gradient methods for unconstrained optimization," Journal of Applied Mathematics and Computing, vol. 58, no. 1, pp. 219-234, 2018, doi: 10.1007/s12190-017-1141-0.

[8] R. Fletcher and C.M. Reeves, "Function minimization by conjugate gradients," The computer journal, vol. 7, no. 2, pp. 149-154, 1964, doi: 10.1093/comjnl/7.2.149.

[9] Y. H. Dai and Y. Yuan, "A nonlinear conjugate gradient method with a strong global convergence property," SIAM Journal on optimization, vol. 10, no. 1, pp. 177-182, 1999, doi: 10.1137/s1052623497318992.

[10] N. Sindhu, P. Kaelo, and M. V. Thuto, "A modified quadratic hybridization of Polak-Ribiere-Polyak and FletcherReeves conjugate gradient method for unconstrained optimization problems," An International Journal of Optimization and Control: Theories \& Applications (IJOCTA) vol. 7, no. 2, pp. 177-185, 2017, doi: 10.11121/ijocta.01.2017.00339.

[11] B. T. Polyak, "The conjugate gradient method in extremal problems," USSR Computational Mathematics and Mathematical Physics, vol. 9, no. 4, pp. 94-112, 1969, doi: 10.1051/m2an/196903r100351.

[12] M. R. Hestenes and E. Stiefel, "Methods of conjugate gradients for solving linear systems," Journal of research of the National Bureau of Standards, vol. 49, no. 1, pp. 409-436, 1952, doi: 10.6028/jres.049.044.

[13] B. A. Hassan, "A new formula for conjugate parameter computation based on the quadratic model," Indonesian Journal of Electrical Engineering and Computer Science, vol. 3, no. 3, pp. 954-961, 2019, doi: 10.11591/ijeecs.v13.i3.pp954-961.

[14] Y. Narushima and H. Yabe, "A survey of sufficient descent conjugate gradient methods for unconstrained optimization," SUT journal of Mathematics, vol. 50, no. 2, pp. 167-203, 2014.

[15] Y. Dai and L. Z. Liao, "New conjugacy conditions and related nonlinear conjugate gradient methods," Applied Mathematics and Optimization, vol. 43, no. 1, pp. 87-101, 2001, doi: 10.1007/s002450010019.

[16] G. Li, C. Tang, and Z. Wei, "New conjugacy condition and related new conjugate gradient methods for unconstrained optimization," Journal of Computational and Applied Mathematics, vol. 202, no. 2, pp. 523-539, 2007, doi: 10.1016/j.cam.2006.03.005.

[17] J. Dong and B. Jiao, "A New Hybrid PRP-DY Conjugate Gradient Method," 2010 Third International Joint Conference on Computational Science and Optimization, 2010, pp. 70-74, doi: 10.1109/CSO.2010.27.

[18] G. Zoutendijk, "Nonlinear programming, computational methods," Integer and nonlinear programming, pp. 37-86, 1970.

[19] A. Zhou, Z. Zhu, H. Fan, and Q. Qing, "Three new hybrid conjugate gradient methods for optimization," Applied Mathematics, vol. 2, no. 3, pp. 303-308, 2011, doi: 10.4236/am.2011.23035.

[20] N. Andrei, "An unconstrained optimization test functions collection," Advanced Modeling and Optimization, vol. 10, no. 1, pp. 147-161, 2008.

[21] K. E. Hillstrom, "A simulation test approach to the evaluation of nonlinear optimization algorithms," $A C M$ Transactions on Mathematical Software (TOMS), vol. 3, no. 4, pp. 305-315, 1977, doi: 10.1145/355759.355760.

[22] B. A. Hassan, A. O. Owaid, and Z. T. Yasen, "A variant of hybrid conjugate gradient methods based on the convex combination for optimization," Indonesian Journal of Electrical Engineering and Computer Science, vol. 20, no. 2 , pp. 1007-1015, 2020, doi: 10.11591/ijeecs.v20.i2.pp1007-1015.

[23] J. Liu and X. Wu, "New three-term conjugate gradient method for solving unconstrained optimization problems," ScienceAsia, vol. 40, pp. 295-300, 2014, doi: 10.2306/scienceasia1513-1874.2014.40.295.

[24] H. N. Jabbar and B. A. Hassan, "Two-versions of descent conjugate gradient methods for large-scale unconstrained optimization," Indonesian Journal of Electrical Engineering and Computer Science, vol. 22, no. 3, pp. 1643-1649, 2021, doi: 10.11591/ijeecs.v22.i3.pp1643-1649.

[25] I. M. Sulaiman, N. A. Bakar, M. Mamat, B. A. Hassan, M. Malik, and A. M. Ahmed, "A new hybrid conjugate gradient algorithm for optimization models and its application to regression analysis," Indonesian Journal of Electrical Engineering and Computer Science, vol. 23, no. 2, pp. 1100-1109, 2021, doi: 10.11591/ijeecs.v23.i2.pp1100-1109. 